\title{
Four QTLs determine crown rust (Puccinia coronata f. sp. lolii) resistance in a perennial ryegrass (Lolium perenne) population
}

\author{
H Muylle ${ }^{1}$, J Baert ${ }^{1}$, E Van Bockstaele ${ }^{1,2}$, J Pertijs ${ }^{3}$ and I Roldán-Ruiz ${ }^{1}$ \\ ${ }^{1}$ Department of Plant Genetics and Breeding, Agricultural Research Centre, Caritasstraat 21, 9090-Melle, Belgium; ${ }^{2}$ Department of Plant \\ Production, University of Ghent, Coupure Links 653, 9000-Gent, Belgium; ' ${ }^{3}$ dvanta Seeds B.V., Vanderhaveweg 2, 4411 RB Rilland, \\ The Netherlands
}

\begin{abstract}
Crown rust resistance is an important selection criterion in ryegrass breeding. The disease, caused by the biotrophic fungus Puccinia coronata, causes yield losses and reduced quality. In this study, we used linkage mapping and QTL analysis to unravel the genomic organization of crown rust resistance in a Lolium perenne population. The progeny of a pair cross between a susceptible and a resistant plant were analysed for crown rust resistance. A linkage map, consisting of 227 loci (AFLP, SSR, RFLP and STS) and spanning $744 \mathrm{cM}$, was generated using the two-way pseudo-testcross approach from 252 individuals. QTL analysis revealed four genomic regions involved in crown rust resistance. Two QTLs were located on LG1 ( $L p P c 4$ and $L p P c 2$ ) and two on LG2 ( $L p P c 3$ and $L p P c 1)$. They explain 12.5, 24.9, 5.5 and $2.6 \%$ of phenotypic variance, respectively. An STS marker,
\end{abstract}

showing homology to $\mathrm{R}$ genes, maps in the proximity of $L p P c 2$. Further research is, however, necessary to check the presence of functional $R$ genes in this region. Synteny at the QTL level between homologous groups of chromosomes within the Gramineae was observed. LG1 and LG2 show homology with group $A$ and $B$ chromosomes of oat on which crown rust-resistance genes have been identified, and with the group 1 chromosomes of the Triticeae, on which leaf rustresistance genes have been mapped. These results are of major importance for understanding the molecular background of crown rust resistance in ryegrasses. The identified markers linked to crown rust resistance have the potential for use in marker-assisted breeding.

Heredity (2005) 95, 348-357. doi:10.1038/sj.hdy.6800729; published online 24 August 2005

Keywords: crown rust; linkage map; Lolium perenne; molecular markers; Puccinia coronata; QTL

\section{Introduction}

Perennial ryegrass (Lolium perenne) is an obligate outbreeding grass species used for either turf or forage purposes. In species grown for their vegetative organs, such as L. perenne, foliar diseases are especially important as they are often correlated with reductions in yield and quality. This is the case for crown rust infections caused by the biotrophic fungus Puccinia coronata (Potter, 1987; Plummer et al, 1990). The severity of crown rust infections can be reduced by good management practices, such as the use of resistant cultivars (Van Bockstaele and Baert, 2004). The use of resistant cultivars is especially needed due to the current tendency to minimize nitrogen input since, the less the nitrogen is applied, the more the crown rust invades ryegrass stands (Kimbeng, 1999). Given the economic impact of crown rust infections, improvement of genetic resistance to this disease is one of the major goals in ryegrass breeding programmes (Wilkins and Humphreys, 2003; Van Bockstaele and Baert, 2004). Currently, breeders identify genotypes carrying resistance genes for crown rust either

Correspondence: H Muylle, Department of Plant Genetics and Breeding, Agricultural Research Centre, Caritasstraat 21, 9090-Melle, Belgium. E-mail: h.muylle@clo.fgov.be

Received 3 February 2005; accepted 18 July 2005; published online 24 August 2005 after natural or artificial infection. A better understanding of the inheritance of the resistance and the identification of the genomic regions that influence resistance can help us to develop more efficient and effective breeding and selection schemes. For example, once identified, the different genomic regions involved in the determination of crown rust resistance can be combined and investigated in a single genotype of positive alleles at several of these loci.

To date, two studies have reported on genomic regions determining crown rust resistance in Lolium. Dumsday et al (2003) detected one major locus (designated LpPc1 by the authors) on linkage group (LG) 2 of the L. perenne genetic map. Muylle et al (2005) detected two clusters of AFLP markers associated with crown rust resistance, explaining $35 \%$ of the variance observed in the segregating population investigated. One cluster mapped to LG 2; the map position of the other cluster was not determined. In both studies, bulked segregant analysis (BSA), followed by mapping of the markers displaying significant associations with the trait, was applied. Although this approach allows the identification of genomic regions that have major effects on the trait, a thorough QTL analysis is necessary to obtain a more general overview of the genetic factors determining resistance and to identify additional genomic regions with minor effects (Quarrie et al, 1999). An 
essential step for QTL analysis is the construction of a detailed linkage map of the population used for phenotypic analysis.

L. perenne displays gametophytic self-incompatibility, controlled by two loci designated $S$ and $Z$ (Thorogood et al, 2002). Linkage analysis in L. perenne is complicated by this self-incompatibility system and by severe levels of inbreeding depression. However, the two-way pseudo-testcross procedure, described by Grattapaglia and Sederoff (1994), can be applied for successful linkage mapping in this outcrossing species. In this procedure, the mapping family consists of an $F_{1}$ progeny derived from a pair-cross between two unrelated, highly heterozygous individuals. This mapping approach has been applied with success in several species (apple, Maliepaard et al, 1998; poplar, Wu et al, 2000), and also in Lolium (Faville et al, 2004; Warnke et al, 2004).

In this study, we first developed a linkage map based on AFLP, SSR, STS and RFLP markers of a L. perenne population segregating for crown rust resistance. Secondly, we used this map to perform QTL analysis. We identified the genomic regions involved in the determination of crown rust resistance in this crop and also studied the syntenic relationships at the genomic and QTL level between Lolium and other grass genomes.

\section{Materials and methods}

\section{Plant materials and phenotypic evaluations}

The mapping population (252 individuals) was derived from a pair cross between two heterozygous perennial ryegrass genotypes, SB2 and TC1, with contrasting phenotypes for crown rust resistance. SB2 was susceptible to crown rust, while TC1 was resistant. The mapping population was evaluated for crown rust resistance at the seedling stage using artificial inoculations, as described in Muylle et al (2005). Scores varied from 1 (= resistant) to 6 (= susceptible). The segregation of the trait indicated that resistance to crown rust in this population was determined by the action of two major loci of which the resistant alleles are dominant and do not interact. It was also demonstrated that additional genomic regions, with smaller individual effects, influence the expression of the trait in this population (Muylle et al, 2005).

\section{Molecular marker analysis}

Before phenotypic evaluation, young leaf material was harvested, frozen in liquid nitrogen, lyophilized and stored under vacuum conditions until used for DNA extraction. Lyophilized material was ground using a mill (Retsch MM200). Genomic DNA was prepared from lyophilized material using a modified CTAB protocol (Weising et al, 1991).

\section{AFLP markers}

Fluorescent AFLP analysis was performed with EcoRI/ MseI and HindIII/MseI enzyme combinations according to Roldán-Ruiz et al (2000). Eight primer combinations (PC) were used for linkage mapping (EcoRI-ACG-MseICAA, EcoRI-AAC-MseI-CAC, EcoRI-ACA-MseI-CAT, EcoRI-AAG-MseI-GCC, EcoRI-ACG-MseI-GGC, HindIIITGG-MseI-CAT, HindIII-TAC-MseI-GAT and HindIIITGC-MseI-GTT).

\section{RFLP markers}

A set of 51 RFLP probes, combined with three restriction enzymes (EcoRI, HindIII and DraI), was used to screen for polymorphisms in the mapping population. The RFLP probes were derived from oat (CDO from Cornell University, USA), rice (RZ from Cornell University, USA; RGR, RGC and RGG from NIAR, Japan), barley (BCD from Cornell University, USA), maize (CSU from Californian State University, USA), wheat (PSR from JIC, UK) and fescue (IBF from AUN, Norway). RFLP analysis was performed using ${ }^{32} \mathrm{P}$-labelled probes as described by Muylle (2003).

\section{SSR markers}

Four sources of SSRs were used: (1) 10 primer pairs from Kubik et al (1999, 2001), (2) 10 primer pairs from Jones et al (2001), (3) 11 primer pairs developed at DvP (Jensen et al, 2005) and (4) 100 unpublished primer pairs developed by Jones et al (La Trobe University, Australia) and licensed to Advanta Van der Have (Rilland, The Netherlands). PCR amplifications of SSR sets (1), (2) and (3) were performed using a Geneamp PCR reagent kit (Applied Biosystems). One primer of each pair was fluorescently labelled to allow fragment separation and detection on an ABI Prism 377 DNA sequencer (PerkinElmer). GS-500 Rox labelled size standard was loaded in each lane to enable automatic analysis of the data. Genescan Analysis Software 2.1 was used to translate the information collected by the ABI377 into fragment sizing information and Genotyper 2.5 software was used to score the fingerprints. The fourth set of SSRs was screened, tested and scored at Advanta Van der Have (Rilland, The Netherlands), using a LiCor Gene ReadIR 4200. The scoring of these SSRs was carried out using Gene ImagIR software (LiCor Inc.).

\section{STS analysis}

A total of 16 primer pairs developed by Lem and Lallemand (2003) were screened for polymorphisms. PCR amplifications were performed using a Geneamp PCR reagent kit (Applied Biosystems). STS fragments were separated on $8 \%$ polyacrylamide gels and visualized by UV illumination after staining with ethidium bromide. The STS fingerprints were scored manually.

We also mapped a marker derived from an RGA sequence, which we PCR-amplified in Lolium using the primer pair s1-NBS1 (Mago et al, 1999). In ryegrass, one fragment of approximately $540 \mathrm{bp}$ displayed homology with known disease-resistance genes. Using the FASTA algorithm, homology was found with the RPP13, $R P P 8, R P M 1$-resistance genes isolated from $A$. thaliana and I2-resistance gene isolated from Lycopersicon esculentum. These genes all have NBS motifs. Sequence information of the amplified fragments from the parents of the mapping population (SB2 and TC1) were used to identify a restriction site polymorphism in order to transform this PCR fragment into a CAPS marker (denominated NBS-1).

\section{Map construction and QTL analysis}

RFLP, SSR and STS markers were scored codominantly if possible. Using this information, six marker classes were defined: (1) a-x--, (2) --xa-, (3) a-xa-, (4) abxab, (5) abxac and (6) abxcd, with the first two characters representing 
the allelic composition of the resistant parent and the last two characters the allelic composition of the susceptible parent ( $a, b, c=$ detectable alleles; - = unknown allele). Class 3 markers were not included in the linkage analysis, as such markers contribute little information to the map and recombination frequency estimates obtained with such markers are typically inaccurate (Maliepaard et al, 1998). The $\chi^{2}$ test integrated in JoinMap 3.0 software (Van Ooijen and Voorrips, 2001) was used to estimate departures from expected segregation ratios. A linkage map was constructed for each parent independently. Initially, distorted markers $(P<0.001)$ were omitted from the analysis. In a second round, distorted markers and markers unmapped during the first step were added to the map only if the map order was not drastically affected. Markers were grouped into linkage groups at LOD equal or higher than 4 using JoinMap 3.0 (Van Ooijen and Voorrips, 2001). Markers of classes (4), (5) and (6) were used as allelic bridges to integrate the parental linkage groups. Marker order was calculated at $\mathrm{LOD}=1.00$ and recombination threshold value (REC) of 0.40 . Map distances were calculated using the Kosambi function.

QTL analysis was carried out with MapQTL 4.0 (Van Ooijen et al, 2002) using automatic cofactor selection and MQM mapping. This analysis was based on the parental linkage maps produced in round 2 in the JoinMap3.0 software, in which markers are not forced to map onto the linkage group.

\section{Comparative analysis}

Comparative mapping was performed as described by Jones et al (2002b). The comparative location of probes in the Triticeae and rice genomes were as reported by Jones et al (2002b) and/or ascertained using the Graingenes (http:// grain.jouy.inra.fr/ggpages/) and Gramene (http://www. gramene.org/) databases.

\section{Results and discussion}

\section{Segregation of DNA markers}

The eight AFLP primer combinations revealed a total of 164 polymorphic markers and included the markers selected in a BSA analysis on the same segregating population that has been reported elsewhere (Muylle et al, 2005). The 29 heterologous RFLP probes polymorphic in the mapping population included both cDNA and genomic DNA probes with known map positions in rice. These markers were included in the $L$. perenne map to enable the study of syntenic relationships between Lolium and other Gramineae (see below). We were able to score the RFLP markers codominantly in 19 cases out of 29 , showing once more the value of RFLP as a codominant marker system. These RFLP probes detected a total of 47 polymorphic loci. Of the 131 SSR primer pairs, 31 (Kubik et al, 1999, 2001; Jones et al, 2002a; Jensen et al, 2005) revealed useful polymorphisms in the mapping population and amplified a total of 54 polymorphic loci. Five STS markers developed by Lem and Lallemand (2003), and the RGA marker developed in this study (NBS-1) were polymorphic in the mapping population and resulted in 10 polymorphic loci useful for linkage analysis.
Overall, $15 \%$ of the markers showed distorted segregation $(P<0.001)$. This figure is in the same order of magnitude as that previously reported in other Lolium studies (Armstead et al, 2002; Jones et al, 2002a, b; Thorogood et al, 2002; Faville et al, 2004; Inoue et al, 2004; Warnke et al, 2004). Apparent segregation distortion can be due to statistical error, genotyping and scoring errors or can be a real biological phenomenon. Genotyping and scoring errors might explain the distorted patterns in our study. In all, 26 of the 275 markers exhibited bias towards the 'presence' allele. For AFLP markers, this can be due to the superimposition on the gels of nonallelic PCR products, corresponding to bands from different loci (ie fragment homoplasy, as has been demonstrated by Vekemans et al (2002) for AFLP markers). A total of 20 AFLP markers deviated towards excess of the 'absence' allele, however, in most of these cases the fragments displayed faint amplification. Possible biological reasons for segregation distortion include the result of pollen tube competition, pollen lethals, preferential fertilization and selective elimination of zygotes (Lu et al, 2002).

\section{Linkage map construction}

Map construction was carried out according to the twoway pseudo-testcross procedure. A total of 275 markers were included in the linkage analysis: $39 \%$ of the markers were derived from the susceptible parent, $42 \%$ of the markers were from the resistant parent and $19 \%$ of the markers were heterozygous in both parents. An integrated map was obtained by aligning the two parental maps on the basis of allelic bridges (Figure 1). This L. perenne genetic map was generated from 252 individuals, spans $744 \mathrm{cM}$, consists of 227 loci grouped into seven linkage groups corresponding to the haploid chromosome number of Lolium. The mean distance between two consecutive loci is $3.28 \mathrm{cM}$. The length of the linkage groups (LGs) vary from 83 till $133 \mathrm{cM}$. The number of markers per linkage group ranges from 17 (LG6) to 48 markers (LG1), with an average of 32 markers per LG. Marker clusters (defined here as $10 \mathrm{cM}$ intervals with more than nine markers) were found in all LG except LG4 and LG6. Major gaps (0 markers / 10 cM) were present in all LG, except LG3; gaps between two adjacent markers exceeding $20 \mathrm{cM}$ were found on LG2, LG6 and LG7.

Using the observed mean chiasma frequency per chromosome of 1.7 obtained from an interspecific cross of L. perenne $\times$ L. multiflorum (Naylor, 1960), the theoretical genome length of Lolium was estimated to be $1190 \mathrm{cM}$. The map obtained here spans $744 \mathrm{cM}$ and thus covers $63 \%$ of the whole genome. Partial genome coverage is also indicated by the substantial number of markers (48 out of 275) that were not mapped into a linkage group. Additional markers, preferrably codominant loci such as SSRs or markers derived from expressed sequences, are needed to integrate these ungrouped markers into the current linkage map.

In total, 23 of the mapped loci deviated significantly from the expected Mendelian segregation ratios. In the present study, a high number of distorted markers were found in LG1 (eight markers) and LG2 (six markers), while the highest percentage of distorted markers was found in LG6, where four out of 18 markers were 


\section{LG1}

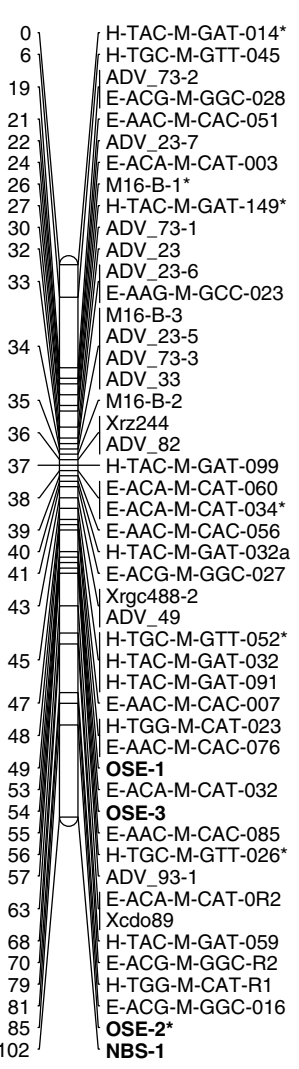

LG3

7) H-TAC-M-GAT-137 $\left.\begin{array}{l}7 \\ 9 \\ 9\end{array}\right) \quad\left[\begin{array}{l}\text { ADV } 10-1 \\ \text { H-TAC-M-GAT-077* } \\ \text { ADV_10-2 }\end{array}\right.$

$20, \|=\mid$ E-AAG-M-GCC-075 $21 \sqrt{ }$ ADV_99-1

25 U $\begin{aligned} & \text { ADV-99-2 } \\ & \text { HAC-M-GAT-022* } \\ & \text { E-AAC-M-CAC-014* }\end{aligned}$

28 E-AAC-M-CAC-088

40 M15-185-2

43 E-AAC-M-CAC-050*

47 E-ACG-M-GGC-106

$\left.\begin{array}{l}50 \\ 51\end{array}\right)$ 值 $\left(\begin{array}{l}\text { XCSU6 } \\ \text { X-TGG-M-CAT-051 } \\ \text { H-AGA-CAT-0R4 }\end{array}\right.$

53 目 $A D V$ - 59-1

月 E-ACG-M-GGC-022

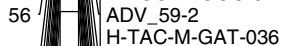

57 E-ACA-M-CAT-0R3

58 Xbcd855-2

59 . E-ACG-M-CAA-079

64 H-TAC-M-GAT-113

68 | E-ACG-M-AA-032

Xdo385
ADV 87

76 E-ACA-M-CAT-039 $\left.\begin{array}{c}0 \\ 21 \\ 28 \\ 33\end{array}\right) / \begin{aligned} & \text { E-ACG-M-CAA-059 } \\ & \text { E-ACG-M-CAA-065 } \\ & \text { uni-001 } \\ & \text { H-TGG-M-CAT-067 } \\ & \text { H-TGC-M-GT-031 }\end{aligned}$

$\left.\left.\begin{array}{l}28 \\ 33\end{array}\right]\right)-\left(\begin{array}{l}\text { H-TGG-M-CAT-067 } \\ \text { H-TGC-M-GTT-03 } \\ \text { YGG }\end{array}\right.$

$\begin{aligned} & 39 \\ & 42 \\ & 4\end{aligned} \mid$ W-ACG-M-CAA-037

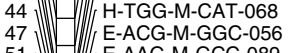

1. 1 . E-AAG-M-GCC-089

1)

1 E-ACA-M-CAT-041

$64)\left(\begin{array}{l}\text { H-TGC-M-GTT-014 } \\ \text { E-AAG-M-GCC-067 }\end{array}\right.$

65 -

$66)\left(\begin{array}{l}\text { E-AAC-M-CAC-041 } \\ \text { H-TAC-M-GAT-061 }\end{array}\right.$

$69)$ E-AAG-M-GCC-007

74 E-ACA-M-CAT-084

78 | E-ACA-M-CAT-O27

(1) E-AAC-M-CAC-079

94
105
E-ACA-M-CAT-043

\section{LG4}

$\left.\begin{array}{l}0 \\ 8\end{array}\right) \quad($ E-ACA-M-CAT-026

E-AAC-M-CAC-112
- 1120

ADV 67
Xpsr104-1

C E-ACA-M-CAT-028

H-TGG-M-CAT-007

- H-TGG-M-CAT-066

1) H-TGG-M-CAT-065

1 - E-AAC-M-CAC-034

48. E-ACA-M-CAT-079

9. E-ACA-M-CAT-071

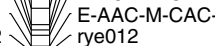

X-AAC-M-CAC-025

H-TGG-M-CAT-118

政 E-ACA-M-CAT-020

$60)$ H X X XO87-M-TGC-M-GTT-036

64 ( Xcsu70

67 Xibf64-2

68 ) $\begin{aligned} & \text { ADV-26-1 } \\ & \mathrm{X} \text { Cdo87-3 }\end{aligned}$

ADV - $26-1$
E-AAG-M-GCC-053
H-TGC-M-GTT-181

94
LPSSRHO1E10-1

\section{LG5}

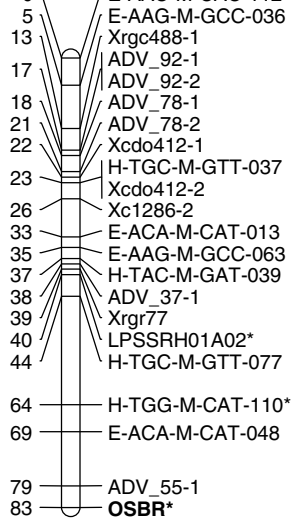

${ }_{83}$ OSBR $^{*}$
LG6

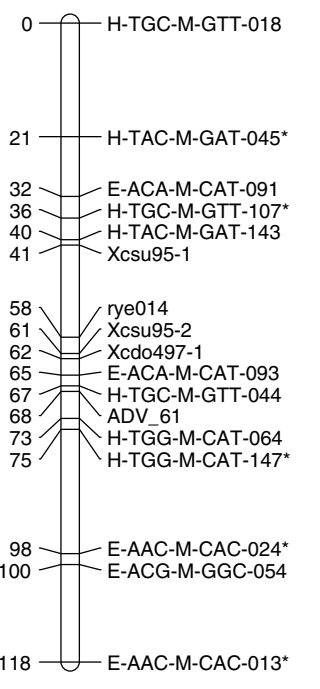

LG7

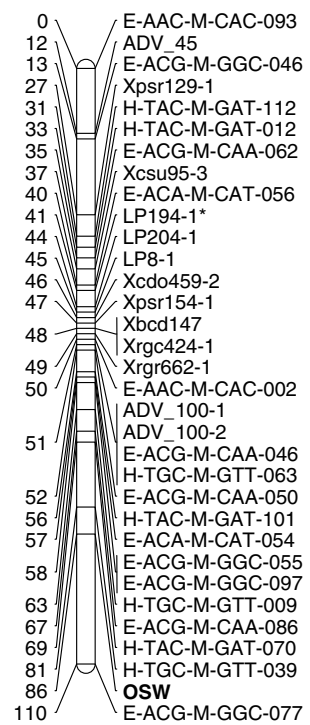

Figure 1 Genetic linkage map for the L. perenne cross based on SSR, RFLP, STS and AFLP markers. RFLPs are shown with prefix X; ESTs are indicated in bold; and SSRs are shown with prefix adv, lp, M, rye or uni; AFLP markers selected during the BSA analysis as being significantly associated with crown rust resistance end with $-\mathrm{Rx}$ (with $\mathrm{x}=$ number). Distorted markers $(P<0.001)$ are marked with *. Linkage groups are numbered according to the ILGI linkage map. 
distorted. LG1 and LG2 represent the chromosomes where the self-incompatibility (SI) loci $S$ and $Z$ have been mapped by Thorogood et al (2002). In LG2, all distorted loci (except rye024_b) mapped in the proximity of the $Z$ locus, which is located in the neighbourhood of BCD135 (http://ukcrop.net/perl/ace/search/FoggDB). Therefore, the presence of a high number of distorted loci in this region can be explained by the SI system present in L. perenne. This phenomenon of distorted segregation ratios of molecular markers due to self-incompatibility has been reported in other species including Fragaria (Sargent et al, 2004), citrus (Ruiz and Asins, 2003), and Phalaris coerulescens (Bian et al, 2004). However, on LG1, just three out of the eight distorted loci mapped close to CDO89 and OSE, markers known to map in the proximity of $S$ (http://ukcrop.net/perl/ace/search/ FoggDB). In LG1 and LG6, distortion can be attributed to the presence of genes related to viability, as also suggested by Thorogood et al (2002) for markers on LG3 in their mapping population. In the Lolium maps published to date, segregation distortion appears in different LGs, indicating that genes that have influence on the generation and survival of the progeny are located at different regions of the genome.

The different marker types used to construct the map were distributed over the different linkage groups. A total of 137 AFLP markers were incorporated to the map. No significant clustering was observed for HindIIIgenerated or EcoRI-generated AFLPs. Although AFLP markers are not very informative in a two-way pseudotestcross, they fulfilled an important role in expanding the genome coverage of the linkage groups (eg in LG6 and LG7). AFLP markers also filled in large gaps between codominant markers (eg LG4).

The AFLP markers identified in the BSA analysis by Muylle et al (2005), with putative associations with crown rust resistance (E-ACA-M-CAT-R3, E-ACA-M-CAT-R4, E-ACG-M-GGC-R2 and H-TGG-M-CAT-R1; Muylle et al, 2005), were also mapped in this study. E-ACA-M-CAT$\mathrm{R} 3$ and E-ACA-M-CAT-R4 mapped in close proximity on LG2; E-ACG-M-GGC-R2 and H-TGG-M-CAT-R1 mapped to LG1. The marker cluster explaining the highest percentage of variation in the crown rust data (E-ACG-M-GGC-R2 and H-TGG-M-CAT-R1) mapped close to the resistance gene analogue, NBS-1.

\section{Alignment with other linkage maps}

The linkage map was aligned with the ILGI (International Lolium Genome Initiative) map (Jones et al, 2002a, b). Alignment was based on 32 SSRs, 10 RFLPs and five STSs in common on both linkage maps. Four inconsistencies (three SSRs and one RFLP) with the ILGI map were observed. These inconsistencies may be due to the detection of multiple loci by the respective SSR primer pairs or RFLP probes. This could certainly be the case for markers ADV87, ADV26 and Xcdo459 as each of these markers detected an extra monomorphic fragment in our mapping population, in addition to the polymorphic mapped fragments.

Although our map was constructed using the two-way pseudo-testcross approach, we could align it with the ILGI map, which was constructed using the one-way pseudo-testcross approach. Marker order was highly conserved and the total map length was in the same order of magnitude. This demonstrates the effectiveness of the two-way pseudo-testcross approach for linkage map construction in outcrossing species. The main advantage of this approach is that the mapping population can be constructed using plants from the breeding pool that show extreme phenotypes in the traits of interest. In this way, the segregation of the traits of interest is ensured in the progeny studied.

\section{QTL analysis}

Phenotypic analysis (Figure 2) of the mapping population revealed two major crown rust-resistance genes (see Muylle et al (2005) for a detailed discussion). To identify the position of genomic regions involved in crown rust resistance, QTL analysis was performed on each parental linkage map (TC1 and SB2) and on the integrated map. Using interval mapping, markers with an LOD score $>3$ associated with crown rust resistance were found on the TC1 (resistant parent) map only and not on the SB2 map. Automatic cofactor selection and MQM analysis on the TC1 map and on the integrated map identified four genomic regions with an LOD score $>5$, on LG 1 and LG2 associated with crown rust resistance (Figure 3). Two QTL regions (QTL1 and QTL2) were the two genomic regions associated with crown rust resistance previously identified by BSA (Muylle et al, 2005). QTL3 and QTL4 represent two novel genomic locations determining crown rust resistance in $L$. perenne. The RGA marker (NBS-1) mapped in the proximity of QTL2. Given the abundance of RGA sequences in plant genomes, many of which are not functional (Leister, 2004; Monosi et al, 2004), the colocation of this RGA marker with QTL2 is not sufficient to conclude that the identified RGA is involved in crown rust resistance. A more detailed study of the identified RGA sequence is necessary before it can be determined whether it is part of a functional gene and whether this gene is involved in crown rust resistance.

Simulations showed that 1-LOD and 1.5-LOD support intervals provide a QTL coverage probability of approximately 90 and 95\%, respectively (Dupuis and Siegmund, 1999). The 1.5-LOD support intervals of the QTLs detected in the integrated map using MQM are given in Table 1.

The proportion of variance explained by the four QTLs detected was estimated using MQM (Table 1). QTL1, QTL2, QTL3 and QTL4 explained 2.6, 24.9, 5.5 and 12.5\% of phenotypic variance, respectively (Table 1). The QTLs

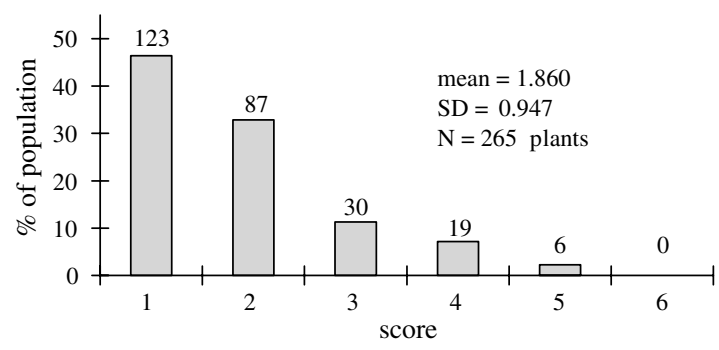

Figure 2 Frequency distribution of mean crown rust scores of the $\mathrm{F}_{1}$ population. Crown rust scores vary from $1=$ resistant to $6=$ susceptible. The number of plants in each category are noted on top of each bar. The population mean, the standard deviation and the total number of plants are also given. 
LG1

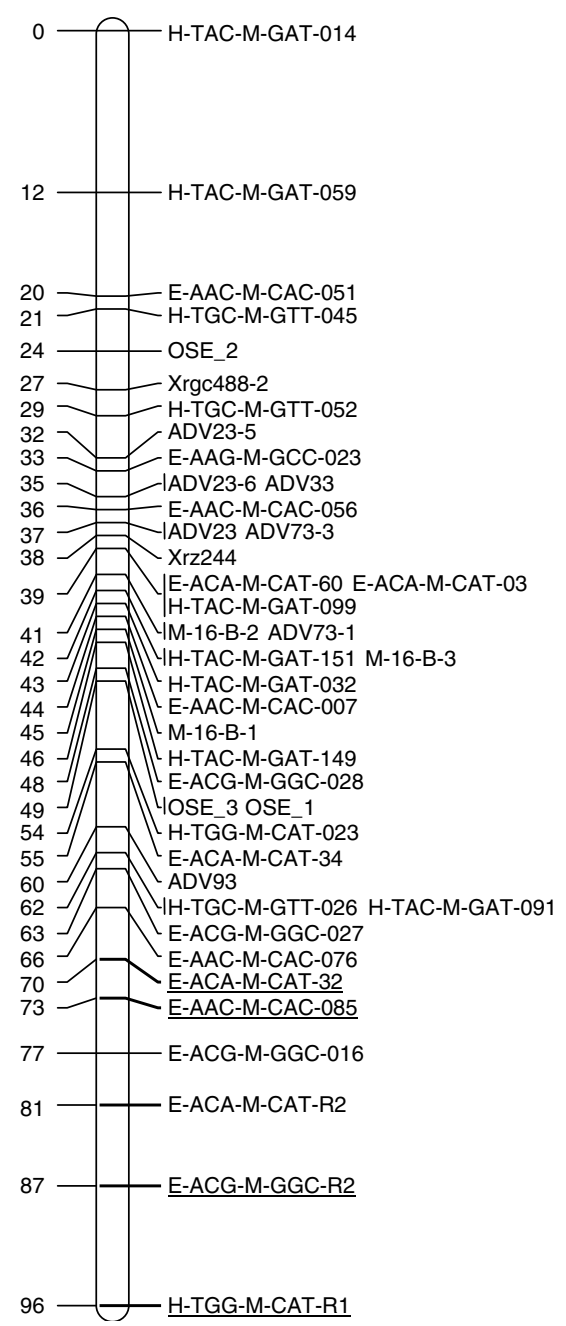

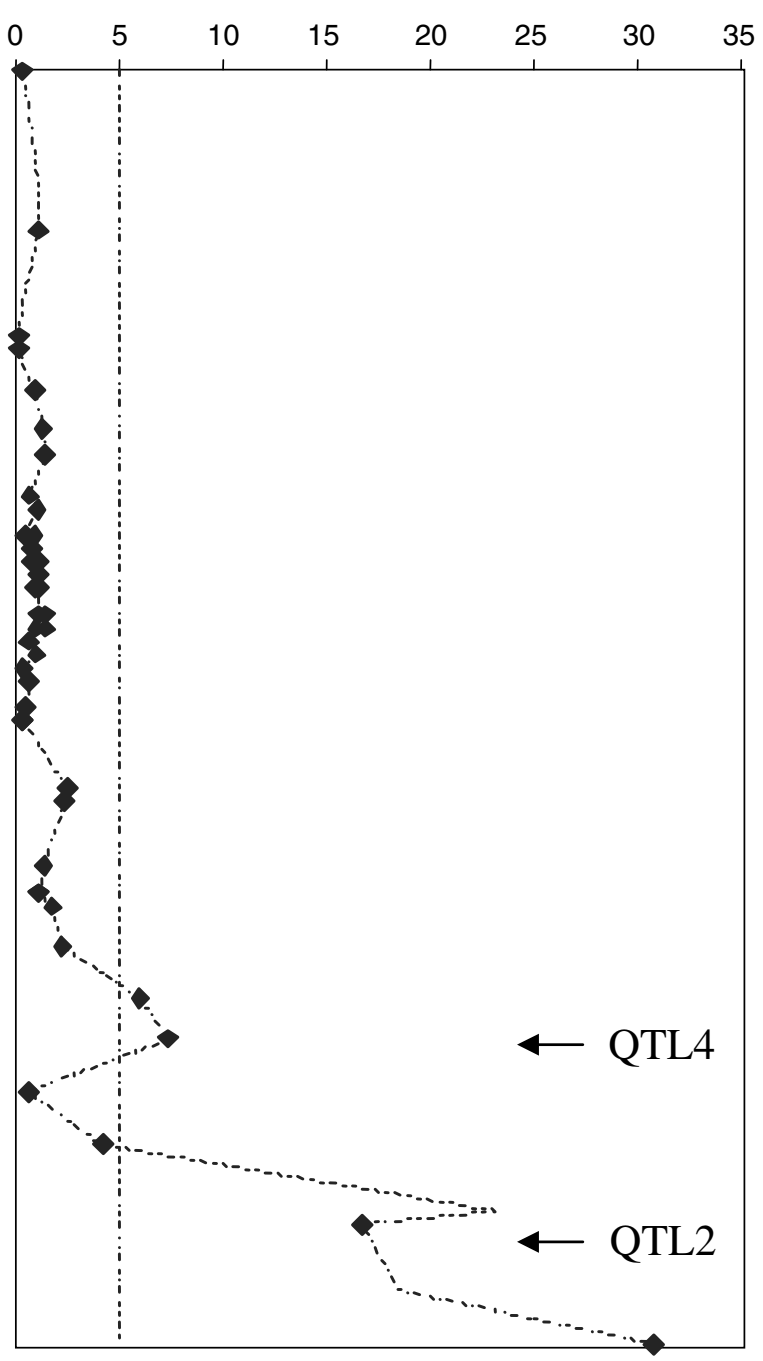

LG2

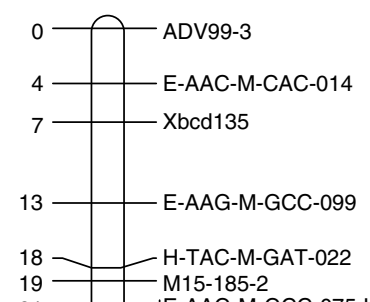

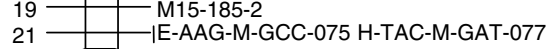
$21 \longrightarrow$ E-AAG-M-GCC-075

27 - H-TGG-M-CAT-051 E-AAC-M-CAC-088 29 - E-ACG-M-GGC-106

31 - X Xdo456

$35-$ H-TAC-M-GAT-036

$37-$ E-AAG-M-GCC-057

$38-$ E-ACG-M-CAA-079 H-TAC-M-GAT-140

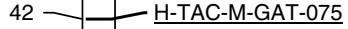

$45-$ E-ACG-M-CAA-032

$48 \longrightarrow$ Xcsub

$52-144-136$

54 E-ACA-M-CAT-39

$57>$ C-AAG-M-GCC-121

587 E-ACG-M-GGC-022

607 E-ACA-M-GGC-022

$64-$ ADV87

66 - X-TGC-M-GTT-055

$68 \sim$ H-TGC-M-GTT-055

\begin{tabular}{l|l}
75 & M4-136-3
\end{tabular}

$78-$ H-TAC-M-GAT-113

$89-$ H-TGC-M-GTT-006

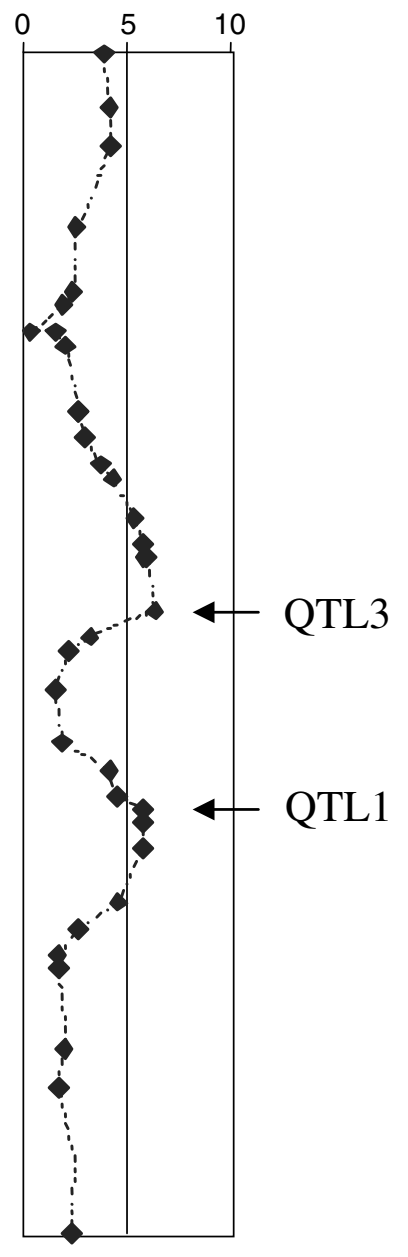

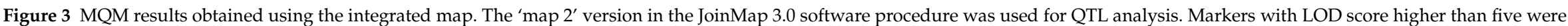
retained for MQM analysis; markers that were selected after MQM are underlined. 
Table 1 1.5 LOD support intervals giving a 95\% probability of QTL location in the integrated map using MQM and the variance explained by each QTL calculated using three different methods: simple linear regression (LR), Interval mapping (IM) and multiple QTL mapping (MQM)

\begin{tabular}{|c|c|c|c|c|c|c|c|c|c|c|}
\hline & \multirow[t]{2}{*}{ MARKER } & \multirow[t]{2}{*}{$L G$} & \multirow{2}{*}{$\begin{array}{l}\text { Position } \\
\quad(c M)\end{array}$} & \multirow{2}{*}{$\begin{array}{l}\text { LOD } \\
\text { score }\end{array}$} & \multirow{2}{*}{$\begin{array}{l}\text { Left border } \\
\text { (cM) }\end{array}$} & \multirow{2}{*}{$\begin{array}{l}\text { Right border } \\
\text { (cM) }\end{array}$} & \multirow{2}{*}{$\begin{array}{c}\text { Size of } 1.5 \\
\text { LOD-interval }(\mathrm{cM})\end{array}$} & \multicolumn{3}{|c|}{ Variance explained } \\
\hline & & & & & & & & $L R(\%)$ & $I M(\%)$ & $M Q M(\%)$ \\
\hline QTL1 & E-ACG-M-GGC-22 & 2 & 58.4 & 5.81 & 55.4 & 65.6 & 10.2 & 3.7 & 6.4 & 2.6 \\
\hline QTL2 & H-TGG-M-CAT-R1 & 1 & 96.1 & 30.76 & 95.2 & - & $0.9^{\mathrm{a}}$ & 20.7 & 23.7 & 24.9 \\
\hline QTL3 & H-TAC-M-GAT-075 & 2 & 42.2 & 6.35 & 24.6 & 43.0 & 18.4 & 9.0 & 10.6 & 5.5 \\
\hline QTL4 & E-AAC-M-CAC-085 & 1 & 72.5 & 7.37 & 70.2 & 73.4 & 3.2 & 7.4 & 13.4 & 12.5 \\
\hline
\end{tabular}

anly the left border was calculated as H-TGG-M-CAT-R1 mapped to the distal end of LG1.

explaining over $20 \%$ of the phenotypic variance, such as QTL2, are strong QTLs. The traits controlled by such QTLs can be considered almost Mendelian and are of extreme interest to breeders for use in selection programmes (Manly and Olson, 1999). QTL pairs were tested for epistatic interactions using GLM analysis (Kover and Caicedo, 2001) but no epistatic effects were identified.

Dumsday et al (2003) detected a major QTL for crown rust resistance on LG2. In our study, QTL1 is also located on LG2, in the proximity of Xcdo385 (Figure 3). According to Jones et al (2002b), Xcdo385 maps to LG2 in the ILGI mapping population. It is in this genomic region that Dumsday et al (2003) also located SSR markers associated with crown rust resistance. This indicates that QTL1 in our study corresponds to the same region as LpPc1 in the study of Dumsday et al (2003). However, QTL1 does not explain a high proportion of variance $(2.6 \%)$, in contrast to the high proportion of variance explained in the study of Dumsday et al (2003). In our study, QTL2 is of major importance ( $>20 \%$ variance explained). Following the nomenclature introduced by Dumsday et al (2003) to designate genomic regions involved in crown rust resistance in L. perenne, it is proposed that this locus is designated $L p P c 2$. Likewise, QTL 1, QTL 3 and QTL 4 can be designated LpPc1, LpPc3 and $L P P c 4$, respectively.

\section{Synteny at the marker and QTL level}

We mapped 31 loci revealed by 29 heterologous RFLP probes. These probes had been previously mapped in rice by Stephenson (1999) and therefore were suitable for comparative mapping. An overview of the location of these probes on the rice map (Stephenson, 1999; gramene database), oat map (Van Deynze et al, 1995a; gramene database), wheat and barley map (Gale et al, 1995; Nelson et al, 1995a, b; Van Deynze et al, 1995b; Marino et al, 1996; gramene database) and the ILGI reference map (Jones et al, 2002b) is given in Table 2 .

Jones et al (2002b) carried out a thorough comparative study between the ILGI map and three other Poaceae maps (Triticeae, rice and oat) on the basis of 109 heterologous probes. Our data provides new additional information for comparative studies. For LG1 of Lolium, we can confirm synteny with LG1 of Triticeae, LG5 of rice, and LGA of oat. For LG2 of Lolium, we have mapped an additional probe (BCD855), which confirms synteny with LG2 of Triticeae, and two probes, which confirm synteny with LG4 and LG7 of rice (CSU6 and BCD855, respectively). In LG3 of Lolium, we mapped two
Table 2 Overview of map positions of heterologous RFLP probes on the rice map (Stephenson, 1999; gramene database), oat map (Van Deynze et al, 1995a), wheat map, barley map (gramene database; Nelson et al, 1995a,b; Van Deynze et al, 1995a; Gale et al, 1995; Marino et al, 1996) and the ILGI map (Jones et al, 2002a)

\begin{tabular}{|c|c|c|c|c|c|c|}
\hline \multirow{2}{*}{$\begin{array}{l}\text { LG in this } \\
\text { study }\end{array}$} & \multirow[t]{2}{*}{ Probe } & \multicolumn{5}{|c|}{$L G$} \\
\hline & & Rice & Oat & Barley & Wheat & ILGI \\
\hline \multirow[t]{3}{*}{1} & RZ244 & $1 / 5$ & $\mathrm{~A} / \mathrm{C}$ & - & $1 \mathrm{~A}$ & - \\
\hline & RGC488 & 10 & - & - & - & - \\
\hline & CDO89 & 5 & $\mathrm{D}$ & - & $1 \mathrm{~A} / 1 \mathrm{D}$ & - \\
\hline \multirow[t]{5}{*}{2} & BCD135 & 4 & B & - & $2 \mathrm{~B} / 4 \mathrm{~A}-$ & 2 \\
\hline & CSU6 & $2 / 4$ & - & - & - & - \\
\hline & BCD855 & 7 & - & - & $2 \mathrm{~A}$ & - \\
\hline & CDO385 & 7 & B & - & - & 2 \\
\hline & CDO456 & 4 & B & - & $2 \mathrm{~A} / 2 \mathrm{~B} / 2 \mathrm{D}$ & 2 \\
\hline \multirow[t]{3}{*}{3} & RGC742 & 1 & - & - & - & - \\
\hline & RGC390 & 8 & - & - & - & 3 \\
\hline & BCD127 & 1 & 一 & - & - & - \\
\hline \multirow[t]{5}{*}{4} & C1286 & 10 & - & $3 \mathrm{H}$ & - & - \\
\hline & PSR104 & 3 & - & $4 \mathrm{H}$ & - & - \\
\hline & CSU25 & 11 & $\mathrm{E}$ & - & $4 B$ & - \\
\hline & CDO87 & 3 & E & - & & - \\
\hline & CSU70 & $1 / 12$ & - & - & - & - \\
\hline \multirow[t]{4}{*}{5} & RGC488 & $6 / 10$ & - & - & - & - \\
\hline & CDO412 & 9 & E & - & $5 \mathrm{~A} / 5 \mathrm{~B} / 5 \mathrm{D}-$ & 5 \\
\hline & RGR77 & $3 / 11 / 12$ & - & - & - & - \\
\hline & C1286 & 10 & - & $3 \mathrm{H}$ & - & - \\
\hline \multirow[t]{2}{*}{6} & CSU95 & $2 / 6$ & - & - & - & - \\
\hline & RGC424 & $2 / 7 / 8$ & G & - & 7 & 6 \\
\hline \multirow[t]{7}{*}{7} & CSU95 & 6 & - & - & - & - \\
\hline & RGC424 & $2 / 7 / 8$ & - & - & - & - \\
\hline & PSR154 & 8 & - & $6 \mathrm{H}$ & - & 7 \\
\hline & BCD147 & 8 & D & - & 3B & 7 \\
\hline & CDO459 & 12 & $\mathrm{~F}$ & - & - & 5 \\
\hline & RGR662 & 8 & - & - & - & - \\
\hline & PSR129 & 6 & - & $7 \mathrm{H}$ & $7 \mathrm{~B}$ & - \\
\hline
\end{tabular}

additional probes (RGC742 and BCD127), which identified synteny with rice LG1. In LG4 of Lolium, we mapped two probes (PSR104 and CDO87), which confirm synteny with LG3 of rice. However, the other three probes mapped in LG4 of Lolium did not show synteny with LG3. Synteny with LGE of oat and LG4 of the Triticeae was identified, but one probe (CDO87) mapped in barley on LG3H. In LG7 of Lolium, we found three additional probes showing synteny with rice LG6 and LG8. One of 
these probes on LG7 of Lolium confirmed synteny with LG7 of the Triticeae.

Although our data set was very small for comparative mapping, it provided additional information to the study made on the basis of the ILGI map. The information obtained from our study includes some confirmation but also some inconsistencies to the results obtained by Jones et al (2002b). Further integration of all available Lolium maps will improve the knowledge about the existence of syntenic relationships between Lolium and other Gramineae.

Many instances of orthology between grasses are reported (reviewed by Devos, 2005). Armstead et al (2004) report on synteny between a major heading-date QTL in perennial ryegrass and the $\mathrm{Hd} 3$ heading-date locus in rice. In addition, we made a comparison between $\mathrm{R}$ genes identified and mapped in other monocots. Several agreements with previous QTL-mapping studies were found. Leaf rust (Puccinia triticina)resistance genes are located on the group 1 chromosomes of the Triticeae species (Van Deynze et al, 1995a). The homologous chromosome in oat, the group A chromosomes, contain resistance genes against Puccinia coronata Cda f. sp. avenae. Yu et al (1996) identified homologous regions for resistance to obligate biotrophs in Avena, Hordeum and Zea mays on the homologous group 1. The group 1 chromosomes of the Triticeae and the group A chromosomes of oat are homologous to the LG1 of Lolium; on this LG, we also found two QTLs (LpPc2 and $L p P c 4)$ linked with crown rust resistance.

Yu and Wise (2000) mapped a cluster of crown rustresistance loci (Pca cluster) to LG B of diploid oat. This LG is homologous to LG2 of the Triticeae and LG2 of Lolium, on which we identified two QTLs (LpPc1 and $L p P c 3$ ) for crown rust resistance.

These findings indicate the presence of homologous regions for resistance genes in the Gramineae. If group 1 and 2 chromosomes of the Triticeae descended from the same chromosomes of a common ancestor, the diseaseresistance loci on them may be orthologous. However, we have very fragmentary data on the synteny between LG1 and LG2 of Lolium and other monocots to make a more detailed synteny analysis for QTLs linked with resistance.

\section{Potential applications}

Although the confidence intervals of the four identified QTLs are large, plant breeders may not need to know the QTL locations with great accuracy if they intend to introgress these regions by marker-assisted selection. Plant breeders are mainly interested in those QTLs, which have a large effect, to incorporate them in elite plants. Marker information can be used to increase the frequency of positive QTLs (and to decrease the frequency of negative QTLs) in these plants. Probably the greatest value of markers in this context is the reduction of linkage drag during introduction of QTLs. Marker information can help to break the unfavourable correlations between quantitative characters of interest. Furthermore, marker information around and within a QTL can be used to develop selection indexes (Kearsey and Farquhar, 1998).

The dissection of a complex trait was carried out using parents with high breeding values and a first products of
QTL analysis of crown rust resistance in perennial ryegrass $\mathrm{H}$ Muylle et al

this study for the breeding programme are resistant genotypes with specific (favourable) QTL configurations. Some questions remain to be answered before the broadscale exploitation of those markers identified as being linked to QTLs with major effects. It is likely that the effect and action of a QTL may vary across environments, across different genetic backgrounds and in response to infection with different spore mixtures. It is, therefore, necessary to test the QTLs identified in this study in different environments with different spore mixtures. An assessment of the diversity of QTL alleles and their action present in a broad genetic population should also be made (Marques et al, 1999; King et al, 2000; Miflin, 2000).

However, taking into account our discovery of synteny, at the QTL level, with other grass species, it is possible that at least some of the QTLs identified in this study represent genomic regions involved in general resistance or genomic regions in which $\mathrm{R}$ genes are clustered (gene-for-gene resistance). Evidence for genefor-gene resistance towards crown rust in Lolium has been reported by several authors (Dumsday et al, 2003; Roderick et al, 2003; Aldaoud et al, 2004). In the case of QTL2 ( $L p P c 2)$, it is likely that we detect a genomic region coding for gene-for-gene resistance as the mapped RGA marker (NBS-1) coincides with $L p P c 2$. It would therefore be interesting to analyse this population for resistance to other diseases affecting Lolium, including bacterial wilt and stem rust and to identify the QTLs which determine resistance to these diseases.

\section{Conclusions}

A linkage map of L. perenne, based on the two-way pseudo-testcross approach, has been constructed for a population derived from two parents with extreme contrasting phenotypes for crown rust resistance. The genetic map spans $744 \mathrm{cM}$ and consists of 227 loci (RFLP, STS, AFLP and SSR), grouped into seven linkage groups. Markers generated with different marker techniques are evenly distributed on the genetic map. The map size $(744 \mathrm{cM})$ is in the range of other published linkage maps of Lolium. The estimated genome coverage is $63 \%$ of the theoretically expected linkage map length for Lolium. The coverage of this map is far from complete as demonstrated by the number of markers that could not be mapped to the linkage groups. Additional markers, particularly codominant and gene-specific markers will help to expand the coverage of the genome in future research.

In this study, four QTLs involved in crown rust resistance have been identified and localized. Two QTLs are located on LG1 ( $L p P c 4$ and $L p P c 2$ ) and two on LG2 ( $L p P c 3$ and $L p P c 1$ ). They explain 12.5, 24.9, 5.5 and $2.6 \%$ of phenotypic variance, respectively. No epistatic interactions were found between these four QTLs.

As reported in previous studies, a high level of synteny between Lolium and the Triticeae was found. Some additional information to the comparative mapping study between Lolium and related species made by Jones et al (2002b) was obtained. Expanding the knowledge of synteny between these species will allow the transfer of information from model species such as rice to less-studied species with larger genomes, like Lolium. 
Mapping of genes or loci with known function are of special interest.

The presence of synteny at the QTL level between homologous groups of chromosomes within the Gramineae was identified. LG1 and LG2 of Lolium show homology with group A and B chromosomes of oat, on which crown rust-resistance genes have been identified. LG1 and LG2 also show homology with the group 1 chromosomes of the Triticeae, on which leaf rustresistance genes have been identified.

\section{Acknowledgements}

We thank Wendy Aartsen, Greet De Smet, Carina Pardon and Ariane Staelens for their technical assistance. We thank dr. ir. J Van Huylenbroeck for his assistance in plant handling. Dr Rindert Peerbolte and Arnd Jan van Wijk (Advanta Seeds BV Rilland, The Netherlands) helped with plant material and fruitful discussions. This research was partially funded by the 'European Gramineae Mapping project' (EGRAM), Contract No B104-CT97-2220. We thank them for support.

\section{References}

Aldaoud R, Anderson MW, Reed KFM, Smith KF (2004). Evidence of pathotypes among Australian isolates of crown rust infecting perennial ryegrass. Plant Breed 123: 395-397.

Armstead IP, Turner LB, Farell M, Skot L, Gomez P, Montoya T et al (2004). Synteny between a major heading date QTL in perennial ryegrass (Lolium perenne L.) and the Hd3 heading date locus in rice. Theor Appl Genet 108: 822-828.

Armstead IP, Turner LB, King IP, Cairns AJ, Humphreys MO (2002). Comparison and integration of genetic maps generated from $\mathrm{F}_{2}$ and $\mathrm{BC}_{1}$-type mapping populations in perennial ryegrass. Plant Breed 121: 501-507.

Bian XG, Friedrich A, Bai JR, Bauman U, Hayman DL, Barker SJ et al (2004). High-resolution mapping of the $S$ and $Z$ loci of Phalaris coerulescens. Genome 47: 918-930.

Devos KM (2005). Updating the 'crop circle'. Curr Opin Plant Biol 8: 155-162.

Dumsday JL, Smith KF, Forster JW, Jones ES (2003). SSR-based genetic linkage analysis of resistance to crown rust (Puccinia coronata f. sp. lolii) in perennial ryegrass (Lolium perenne). Plant Pathol 52: 628-637.

Dupuis J, Siegmund D (1999). Statistical methods for mapping quantitative trait loci from a dense set of markers. Genetics 151: 373-386.

Faville MJ, Vecchies AC, Schreiber M, Drayton MC, Hughes LJ, Jones ES et al (2004). Functionally associated molecular genetic marker map construction in perennial ryegrass (Lolium perenne L.). Theor Appl Genet 110: 12-32.

Gale MD, Atkinson MD, Chinoy CN, Harcourt RL, Jia J, Li QY et al (1995). Genetic maps of hexaploid wheat. In: Li ZS, Xin ZY (eds) Proceedings of the 8th International Wheat Genetics Symposium. China Agricultural Scientech Press: Beijing, China, pp 29-40.

Grattapaglia D, Sederoff R (1994). Genetic linkage maps of Eucalyptus grandis and Eucalyptus urophylla using a pseudotestcross: mapping strategy and RAPD markers. Genetics 137: 1121-1137.

Inoue M, Gao Z, Hirato M, Fujimori M, Cai H (2004). Construction of a high-density linkage map of Italian ryegrass (Lolium multiflorum Lam.) using restriction fragment length polymorphism, amplified fragment length polymorphism, and telomeric repeat associated sequence markers. Genome 47: 57-65.

Jensen LB, Muylle $\mathrm{H}$, Arens P, Andersen CH, Holm PB, Ghesquiere $M$ et al (2005). Development and mapping of a public reference set of SSR markers in Lolium perenne L. Mol Ecol Notes (online publication date 19-7-05; doi:10.111/j.14718286.2005.01043.X)

Jones ES, Dupal MP, Dumsday JL, Hughes LJ, Forster JW (2002a). An SSR-based genetic linkage map for perennial ryegrass (L perenne L.). Theor Appl Genet 105: 577-584.

Jones ES, Dupal MP, Kölliker R, Drayton MC, Foster JW (2001). Development and characterization of simple sequence repeat (SSR) markers for perennial ryegrass (Lolium perenne L.). Theor Appl Genet 102: 405-415.

Jones ES, Mahoney NL, Hayward MD, Armstead IP, Jones JG, Humphreys MO et al (2002b). An enhanced molecular marker based genetic map of perennial ryegrass (Lolium perenne) reveals comparative relationships with other Poaceae genomes. Genome 45: 282-295.

Kearsey MJ, Farquhar AGL (1998). QTL analysis in plants; where are we now? Heredity 80: 137-142.

Kimbeng CA (1999). Genetic basis of crown rust resistance in perennial ryegrass, breeding strategies, and genetic variation among pathogen populations: a review. Aust J Exp Agr 39: 361-378.

King GJ, Maliepaard C, Lynn JR, Alston FH, Durel CE, Evans $\mathrm{KM}$ et al (2000). Quantitative genetic analysis and comparison of physical and sensory descriptors relating to fruit flesh firmness in apple (Malus pumila Mill). Theor Appl Genet 100: 1074-1084.

Kover PX, Caicedo AL (2001). The genetic architecture of disease resistance in plants and the maintenance of recombination by parasites. Mol Ecol 10: 1-16.

Kubik C, Meyer WA, Gaut BS (1999). Assessing the abundance and polymorphism of simple sequence repeats in perennial ryegrass. Crop Sci 39: 1136-1141.

Kubik C, Sawkins M, Meyer WA, Gaut BS (2001). Genetic diversity in seven perennial ryegrass (Lolium perenne L.) cultivars based on SSR markers. Crop Sci 41: 1565-1572.

Leister D (2004). Tandem and segmental gene duplication and recombination in the evolution of plant disease resistance gene. Trends Genet 20: 116-122.

Lem P, Lallemand J (2003). Grass consensus STS markers: an efficient approach for detecting polymorphisms in Lolium. Theor Appl Genet 107: 1113-1122.

Lu H, Romereo-Severson J, Bernardo R (2002). Chromosomal regions associated with segregation distortion in maize. Theor Appl Genet 105: 622-628.

Mago R, Nair S, Mohan M (1999). Resistance gene analogues from rice: cloning, sequencing and mapping. Theor Appl Genet 99: 50-57.

Maliepaard C, Alston FH, Van Arkel G, Brown LM, Chevreau E, Dunemann F et al (1998). Aligning male and female linkage maps of apple (Malus pumila Mill) using multi-allelic markers. Theor Appl Genet 97: 60-73.

Manly KF, Olson JM (1999). Overview of QTL mapping software and introduction to Map Manager QT. Mamm Genome 10: 327-334.

Marino CL, Nelson JC, Lu YH, Sorrells ME, Leroy P, Tuleen NA et al (1996). Molecular genetic maps of the group 6 chromosomes of hexaploid wheat (Triticum aestivum L. em. Thell). Genome 39: 359-366.

Marques CM, Vasquez-Kool J, Carocha VJ, Ferreira JG, O'Malley DM, Liu B-H et al (1999). Genetic dissection of vegetative propagation traits in Eucalyptus tereticornis and E. globules. Theor Appl Genet 99: 936-946.

Miflin B (2000). Crop improvement in the 21st century. J Exp Bot 51: $342,1-8$..

Monosi B, Wisser RJ, Pennill L, Hubert SH (2004). Full-genome analysis of resistance gene homologues in rice. Theor Appl Genet 109: 1434-1447.

Muylle H (2003). Genetic Dissection of Crown Rust (Puccinia coronata) Resistance in Ryegrasses (Lolium spp.) Using Molecular Markers. PhD thesis, University of Ghent, 161pp. 
Muylle H, Baert J, Van Bockstaele E, Roldán-Ruiz I (2005). Identification of molecular markers linked with crown rust (Puccinia coronata f. sp. lolii) resistance in perennial ryegrass (Lolium perenne) using AFLP markers and a bulked segregant approach. Euphytica (in press).

Naylor B (1960). Species differentiation in the genus Lolium. Heredity 15: 219-233.

Nelson JC, Van Deynze AE, Autrique E, Sorrells ME, Lu YH, Merlino $M$ et al (1995a). Molecular mapping of wheat homologous group 2. Genome 38: 516-524.

Nelson JC, Van Deynze AE, Autrique E, Sorrells ME, Lu YH, Negre $S$ et al (1995b). Molecular mapping of wheat homologous group 3. Genome 38: 525-533.

Plummer R, Hall R, Watt T (1990). The influence of crown rust (Puccinia coronata) on tiller production and survival of perennial ryegrass (Lolium perenne) plants in simulated swards. Grass Forage Sci 45: 9-16.

Potter LR (1987). Effect of crown rust on regrowth competitive ability and nutritional quality of perennial and Italian ryegrass. Plant Pathol 36: 455-461.

Quarrie S, Lazić-Jančić V, Kovačević D, Steed A, Pekić S (1999). Bulk segregant analysis with molecular markers and its use for improving drought resistance in maize. J Exp Bot 337: 1299-1306.

Roderick HW, Morgan WG, Harper JA, Thomas HM (2003). Introgression of crown rust (Puccinia coronata) resistance from meadow fescue (Festuca pratensis) into Italian ryegrass (Lolium multiflorum) and physical mapping of the locus. Heredity 91: 396-400.

Roldán-Ruiz I, Dendauw J, Van Bockstaele E, Depicker A, De Loose M (2000). AFLP markers reveal high polymorphic rates in ryegrasses (Lolium spp.). Mol Breed 6: 125-134.

Ruiz C, Asins MJ (2003). Comparison between Poncirus and Citrus genetic linkage maps. Theor Appl Genet 106: 826-836.

Sargent DJ, Davis TM, Tobutt KR, Wilkinson MJ, Battey NH, Simpson DW (2004). A genetic linkage map of microsatellite, gene-specific and morphological markers in diploid Fragaria. Theor Appl Genet 109: 1385-1391.

Stephenson P (1999). Second Year Report of the European Gramineae mapping project (EGRAM), contract NO BI04 CT97 2220.

Thorogood D, Kaiser WJ, Jones JG, Armstead I (2002). Selfincompatibility in ryegrass 12 genotyping and mapping the $S$ and $Z$ loci of L. perenne. Heredity 88: 385-390.
Van Bockstaele E, Baert J (2004). Improvement of perennial ryegrass (L. perenne). Plant Sci 16: 483-488.

Van Deynze AE, Dubcovsky J, Gill KS, Nelson JC, Sorrells ME, Dvorak J et al (1995a). Molecular-genetic maps for group 1 chromosomes of Triticeae species and their relation to chromosomes in rice and oat. Genome 38: 45-59.

Van Deynze AE, Nelson JC, O'Donoughue LS, Ahn SN, Siripoonwiwat W, Harrington SE et al (1995b). Comparative mapping in grasses oat relationships. Mol Genet Genomics 249(3): 349-356.

Van Ooijen JW, Boer MP, Jansen RC, Maliepaard C (2002). MapQTL ${ }^{(\mathbb{R}}$ 4.0, Software for the Calculation of QTL Positions on Genetic Maps. Plant Research International: Wageningen, the Netherlands.

Van Ooijen JW, Voorrips RE (2001). Joinmap ${ }^{\circledR}$ 3.0, Software for the Calculation of Genetic Linkage Maps. Plant Research International: Wageningen, The Netherlands.

Vekemans X, Beauwens R, Lemaire M, Roldán-Ruiz I (2002). Data from amplified fragment length polymorphism (AFLP) markers show indication of size homoplasy and of a relationship between degree of homoplasy and fragment size. Mol Ecol 11: 139-151.

Warnke SE, Barker RE, Jung G, Sim SC, Rouf Mian MA Saha MC et al (2004). Genetic mapping of an annual $\mathrm{x}$ perennial ryegrass population. Theor Appl Genet 109: 294-304.

Weising K, Beyermann B, Ramser J, Kahl G (1991). Plant DNA fingerprinting with radioactive and digoxigenated oligonucleotide probes complementary to simple repetitive DNA sequences. Electrophoresis 12: 159-169.

Wilkins P, Humphreys MO (2003). Progress in breeding perennial forage grasses for temperate agriculture. J Agr Sci 140: 129-150.

Wu RL, Han YF, Hu JJ, Fang JJ, Li L, Li ML et al (2000). An integrated genetic map of Populus deltoids based on amplified fragment length polymorphisms. Theor Appl Genet 100: 1249-1256.

Yu GX, Wise RP (2000). An anchored AFLP- and retrotransposon-based map of diploid Avena. Genome 43 736-749.

Yu YG, Buss GR, Maroof MAS (1996). Isolation of a superfamily of candidate disease-resistance genes in soybean based on a conserved nucleotide-binding site. Proc Natl Acad Sci USA 93 : 11751-11756. 